\title{
Modeling Weak Discontinuities in Saturated Porous Medium by Using Extended Finite Element Method
}

\author{
E. Mikaeili, and M.T. Kazemi
}

\begin{abstract}
The present paper discusses the development of the modeling coupled deforming porous media which contains weak discontinuity. The formulation is based on the coupled solid deformation and fluid pressure. The spatial and time discretization is carried out by extended finite element method and generalized Newmark method, respectively. The fluid flow normal to material discontinuity is modeled by enhancing the pressure field with the modified level set function, which is mostly used for weak discontinuities. Finally as a verification, a problem of material interface under the assumption of fully coupled system is examined.
\end{abstract}

Index Terms - XFEM - Coupled Porous Media - Weak Discontinuity.

\section{INTRODUCTION}

Porous materials like soil, are compound of void that are in connection with each other. In fully saturated case, the voids are filled with fluid, in which leads to complex interaction between the phases i.e. solid and fluid phases. The fundamental theories of porous media, averaging theories were introduced by Terzaghi [1] and Biot [2]. The plasticity state of saturated porous media was presented by Boer and Kowalski [3] and later Zeinkiewicz and Shiomi [4] proposed a nonlinear algorithm of elasto-plasticity in deformable porous media.

The finite element method is a robust and sufficient method in which is employed to many engineering problems, however it has the drawback of modeling discontinuities. Modeling stationary discontinuities requires refined meshing in order to reach the enough accuracy in capturing the singular fields near the discontinuity. Also modeling moving discontinuities such as crack propagation, shear banding [5] and phase changing is tractable only when updating the mesh arrangement in each time step as to conform to the line discontinuity.

In order to capture the arbitrarily discontinuities, based on the concept of partition of unity[6,7] and generalized finite element method[8,9], the local enrichment of standard finite element solutions presented by Moes et al [10] and Belytchko and Black [11], that later called as extended finite element method.

This method alleviates the difficulty of remeshing the solution domain proposed by FEM, significantly enhances the numerical solution by saving the computation time.

In current study, the XFEM [12] is used to model the weak discontinuity by discretizing the weak form of governing

E. Mikaeili, and M.T. Kazemi Department of Civil Engineering, Sharif university of Technology, equations, i.e. linear momentum balance equation and mass conservation equation. Lastly a numerical example is presented to support the proposed theory, which is compared with XEFG model [13].

\section{PROCEDURE FOR PAPER SUBMISSION}

In this section, the governing equations for saturated porous media is presented. The momentum balance equation can be written as follows:

$\nabla . \sigma+\rho_{t} B=0$

Where $B$ is the body force and $\rho_{t}$ is the total density of the media, and can be formulated as,

$\rho_{t}=(1-n) \rho_{s}+n \rho_{f}$

Where $\rho_{s}$ and $\rho_{f}$ are the density of solid and fluid phase and $\mathrm{n}$ denotes the porosity. The total stress vector $\sigma$ can be written in terms of the effective stress vector $\sigma^{\prime}$,

$\sigma=\sigma^{\prime}-\alpha m\left(\mathrm{p}_{f}\right)$

And the constitutive relation is defined in terms of the effective stress and strain increments,

$d \sigma^{\prime}=C(d \varepsilon)$

Where $C$ is the elastic stiffness tensor and $\alpha$ is the Biot's constant,

$\alpha=1-\frac{K_{T}}{K_{S}}$

With $K_{T}$ and $K_{S}$ defined as the bulk modulus of porous skeleton and solid grains, respectively. The momentum balance relation for the water phase leads to the generalized form of the Darcy equation,

$v^{d}=\frac{K}{\mu_{f v}}\left[-\nabla \mathrm{p}_{f}+\rho_{f} B\right]$

(6)

And the final form of the continuity equation for the water phase can be expressed as

$\left[\frac{(\alpha-n)}{K_{S}}+\frac{n}{K_{f}}\right] \frac{\partial \mathrm{p}_{f}}{\partial t}+\alpha \nabla \cdot v_{s}$

$+\nabla \cdot\left[K\left(-\nabla \mathrm{p}_{f}+\rho_{f}(B)\right)\right]=0$

Where $K$ is the permeability tensor, $K_{f}$ and $v_{s}$ are defined as the bulk modulus and the Darcy velocity for the water phase, 
respectively. The boundary conditions include the imposed tractions and water fluxes on the outward boundaries and the corresponding prescribed essential boundary conditions for solid displacements and water pressures,

$$
\begin{aligned}
& u=\tilde{u} \\
& \mathrm{p}_{f}=\tilde{\mathrm{p}}_{f}
\end{aligned}
$$

By applying the weighted residual method to equations, (1) and (7), with their corresponding boundary conditions, equations (8). The final weak form of equations will be obtained.

$$
\begin{aligned}
& \int_{\Omega} \delta\left(\mathrm{L}_{u} u\right)^{T}(\sigma) d \Omega-\int_{\Omega} \delta u^{T} B d \Omega-\int_{\Gamma_{T}} \delta u^{T} T d \Gamma_{t}=0 \\
& \int_{\Omega} \delta\left(\mathrm{p}_{f}\right)^{T} \cdot\left[\frac{(\alpha-n)}{K_{S}}+\frac{n}{K_{f}}\right] \frac{\partial \mathrm{p}_{f}}{\partial t} d \Omega-\int_{\Omega} \delta\left(\mathrm{p}_{f}\right)^{T} \alpha m^{T} L_{u} \frac{\partial u}{\partial t} d \Omega(10) \\
& -\int_{\Omega} \delta\left(\mathrm{L}_{p f} \mathrm{p}_{f}\right)^{T} \cdot\left[K\left(-\nabla \mathrm{p}_{f}+\rho_{f}(B)\right)\right] \mathrm{d} \Omega=0
\end{aligned}
$$

$\delta u$ and $\delta p_{f}$ represent the variations of solid displacement and water pressure, respectively and act as the weighting functions in the proposed Galerkin weak form of the fully coupled saturated porous medium. The differential operators $D_{u}$ and $D_{p}$ are defined as,

$$
D_{u}=\left[\begin{array}{ll}
\frac{\partial}{\partial x} & 0 \\
0 & \frac{\partial}{\partial y} \\
\frac{\partial}{\partial y} & \frac{\partial}{\partial x}
\end{array}\right], \quad D_{p}=\left[\begin{array}{c}
\frac{\partial}{\partial x} \\
\frac{\partial}{\partial y}
\end{array}\right]
$$

\section{XFEM DISCRETIZATION}

In order to model the displacement and pressure field, the following enriched variables are defined;

$$
\begin{aligned}
& P(x, t)=\sum_{s} N_{p s}(x) \bar{P}_{s}(\mathrm{t})+\sum_{d} N_{p d}(x)\left(\chi(x)-\chi_{d}(x)\right) \overline{\mathrm{a}}_{d}(t) \\
& \mathrm{U}(\mathrm{x}, \mathrm{t})=\sum_{s} N_{u s}(x) U(t)
\end{aligned}
$$

where $N_{u s}(x)$ and $N_{p}^{s t d}(x)$ stand for the standard finite element shape functions for discretization of the solid displacements and water pressures, respectively. $\bar{P}_{s}(\mathrm{t})$ and $\overline{\mathrm{a}}_{d}$ are the standard and enrichment degrees of freedom of pressure field. $\chi(\mathrm{x})$ is the modified level set function (14) representing the gradient discontinuity part of the pressure field.

$$
\chi(\mathrm{x})=\sum_{s} N_{p s}(x)\left|\Phi_{s}\right|-\left|\sum_{d} N_{p s}(x) \Phi_{s}\right|
$$

Insertion of the enriched approximation $(12,13)$ into the weak form equations of (9) and (10) results in the complete discretized set of constrained equations for the fully coupled saturated deforming porous medium,

$$
\begin{aligned}
& \int_{\Omega} B^{T, n+1} \sigma^{\prime} d \Omega-Q_{s f}^{n+1} \mathrm{p}_{f}^{n+1}=F_{u}^{n+1} \\
& Q_{f s}^{n+1} \dot{U}^{n+1}+p_{f f}^{n+1} \dot{p}_{f}^{n+1}+H_{f f}^{n+1} p_{f}^{n+1}=F_{f}^{n+1}
\end{aligned}
$$

Where,

$$
\begin{aligned}
& K=\int_{\Omega} B_{u}^{T} C B_{u} d \Omega \\
& Q_{s f}=\int_{\Omega} B_{u}^{T} \alpha m N_{p f} d \Omega \\
& Q_{f s}=\int_{\Omega} N_{p f}^{T} \alpha m^{T} \cdot B_{u} d \Omega \\
& P_{f f}=\int_{\Omega} N_{p f}^{T} \cdot\left(\frac{1}{Q_{f f}}\right) \cdot N_{p f} d \Omega \\
& H_{f f}=\int_{\Omega}\left(L_{p f} N_{p f}\right)^{T} K\left(\mathrm{~L}_{p f} N_{p f}\right) \\
& B_{u=}\left[B_{u}^{s t d}\right], N_{u}=\left[N_{u}^{s t d}\right], \\
& N_{p f}=\left[N_{p f}^{s t d} N_{p f}^{a b s}\right]
\end{aligned}
$$

And using the Newton-Raphson algorithm, the final form of the discretized set of equations can be extracted as,

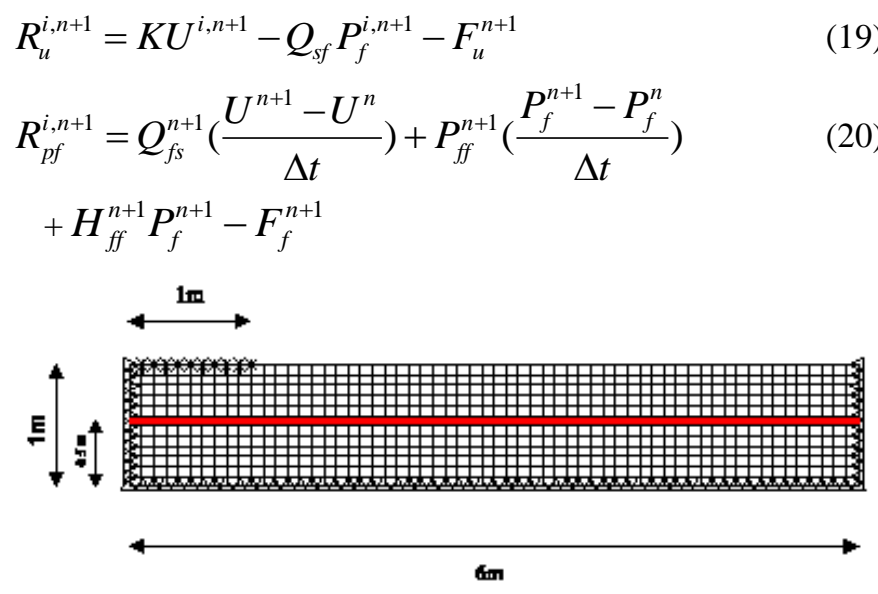

Fig. 1. Meshing of Fully Saturated one And Double Layered Porous Media

And the residual and unknown vectors can be expressed by:

$R^{i, n+1}=\left\{\begin{array}{l}R_{u}^{i, n+1} \\ R_{p f}^{i, n+1}\end{array}\right\}, X^{i, n+1}=\left\{\begin{array}{l}U^{i, n+1} \\ P_{f}^{i, n+1}\end{array}\right\}$

Differentiating the residual with respect to the unknowns vector $\mathrm{X}$, gives the Jacobian matrix for the system of equations,

$$
J=\left[\begin{array}{ll}
K & -Q_{s f} \\
\frac{Q_{f s}}{\Delta t} & \frac{P_{f f}}{\Delta t}+H_{f f}
\end{array}\right]
$$


The following fully coupled system of equations should then be solved at each time step to compute the vector of unknown increments,

$$
R^{i+1, n+1}=R^{i, n+1}+J\left[d X^{i+1, n+1}\right]=0
$$

In which $R^{i, n+1}$ is given in (21), (22) and is computed as following:

$R^{i, n+1}=J\left[\begin{array}{l}U^{i, n+1} \\ P_{f}^{i, n+1}\end{array}\right]-\left[\begin{array}{c}F_{u} \\ F_{f}\end{array}\right]-P^{n}$

In above equation, variable $P^{n}$ is defined as the value of converged solution from the last time step of analysis:

$$
P^{n}=\left[\begin{array}{c}
0 \\
Q_{f s}\left(\frac{U^{n}}{\Delta t}\right)+P_{f f}\left(\frac{P_{f}^{n}}{\Delta t}\right)
\end{array}\right]
$$

Then, the unknowns vector is updated at each iteration until the required convergence criterion is satisfied:

$$
\left[\begin{array}{l}
U^{i+1, n+1} \\
P_{f}^{i+1, n+1}
\end{array}\right]=\left[\begin{array}{l}
U^{i, n+1} \\
P_{f}^{i, n+1}
\end{array}\right]+\left[\begin{array}{l}
d U^{i+1, n+1} \\
d P_{f}^{i+1, n+1}
\end{array}\right]
$$

\section{NUMERICAL RESULTS}

\section{1) One Layer Saturated Porous Media}

A consolidation analysis of a saturated porous media is presented to qualify the finite element model accuracy. The proposed model geometry and boundary conditions as shown in Fig. 1 are the same as the model of double layer porous media. And the mechanical properties of the media, are similar to the upper sub layer of the following example, as presented in table. 1. The consolidation at the surface of centerline, Fig. 2 and the variation in pressure at mid-height of centerline, Fig. 3 are presented and compared with Samimi and Pak [14].

\section{2) Double layer saturated porous media}

In order to testify the utility of the proposed method, a double layered porous media is loaded according to the Fig. 1. The media specimen is $1 \mathrm{~m}$ and $6 \mathrm{~m}$ width with finite boundary to eliminate the effects of the infinite boundary problems and the modeled geometry is half of real one as a result of symmetry of loading to decrease the calculation time. The mechanical properties of two layers are presented in table. 1 .

TABLE 1

MATERIAL PROPERTIES

\begin{tabular}{|l|c|}
\hline$k$ (Intrinsic permeability), upper sub layer & $10^{-7} \mathrm{~m}^{2}$ \\
\hline$k$ (Intrinsic permeability), lower sublayer & $\left(10^{-7} / 4\right) \mathrm{m}^{2}$ \\
\hline$E$ (Young's Modulus), upper sub layer & $100 \mathrm{KPa}$ \\
\hline$E$ (Young's Modulus), lower sub layer & $200 \mathrm{KPa}$ \\
\hline$\mu_{f}$ (Fluid Viscosity) & $10^{-3} \mathrm{~Pa} . \mathrm{s}$ \\
\hline$\Upsilon_{w}$ (Specific Weight of Water) & $10 \mathrm{KP}$ \\
\hline$U$ (Poisson's Ratio) & 0.3 \\
\hline
\end{tabular}

The materials are different in stiffness and permeability. In which the Young's modulus and permeability of lower layer is double and a quarter of upper layer respectively. The other material properties are equal for whole the media. The natural boundary conditions are applied as follows: a constant strip load of $1000 \mathrm{KN}$ to the length of $1 \mathrm{~m}$ applied to the top edge of the media as shown in Fig. 1. and the drainage is permitted only at the top edge while the others are assumed to be impermeable. The constraints applied to the model is limiting the displacement in $\mathrm{x}$ direction for the right, left and bottom edges and the bottom edge for $y$ direction.

The material interface imposes a weak discontinuity in pore pressure field. In which there is a jump in the gradient of pressure normal to the discontinuity line. The gradient of pressure in normal direction across the height of sample is plotted in Fig. 5. for last time step. The results are compared with the Goudarzi and Mohammadi [13] that is based on XEFG method. As shown in Fig. 4. the discontinuity in gradient of pressure (in other words the discontinuity in fluid flow) vanishes as getting far from the loading points. In fact this is the consequence of lack fluid flow in far field. Finally the variation of pore pressure along the height of the media in centerline is computed as in Fig. 6. in which displays acceptable accordance with the comparing model [13].

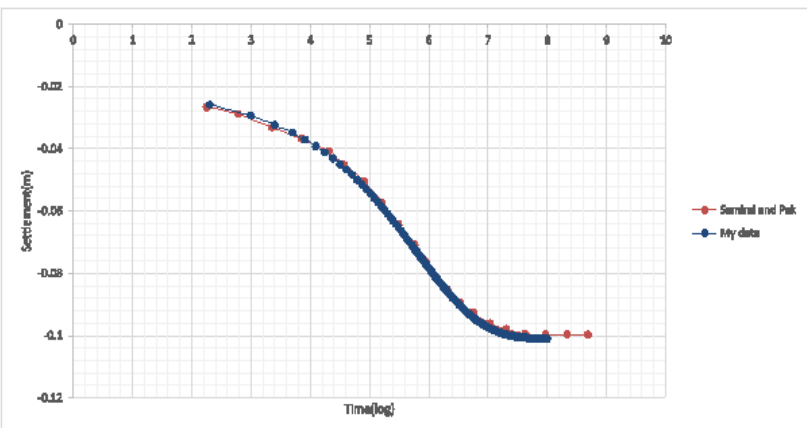

Fig. 2. Settlements At The Surface Of Centerline

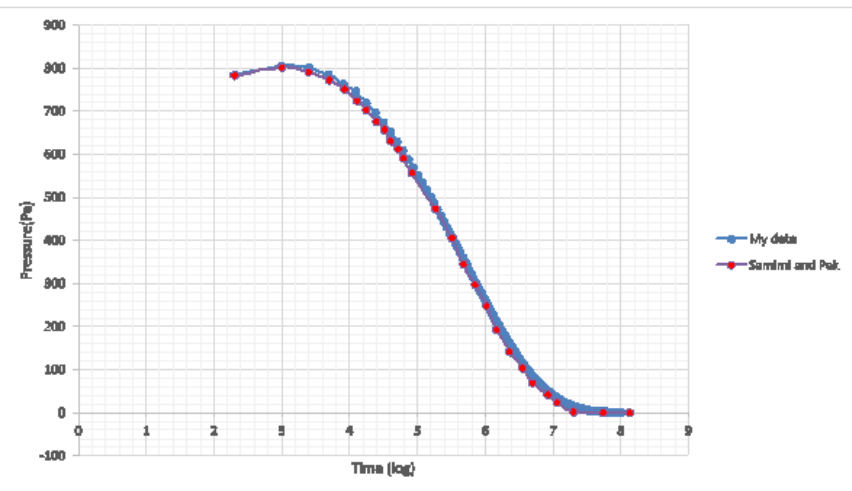

Fig. 3. Water Pressure Distribution At Mid-Height Of Centerline 


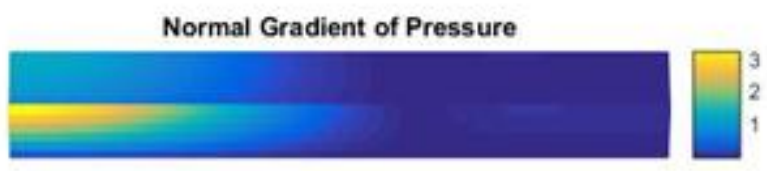

Fig. 4. Values of Normal Gradient of Pressure at Integration Points $(\mathrm{KPa} / \mathrm{m})$

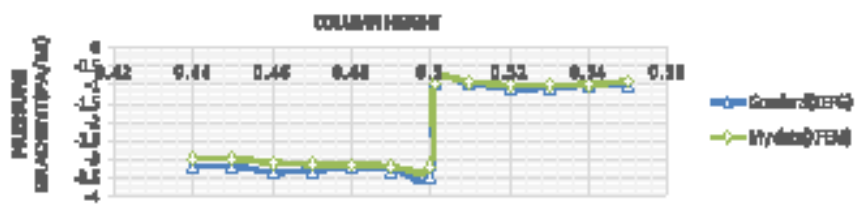

Fig. 5. Variations Of Water Pressure Gradients Along The Soil Height For The Last Time Step Of The Analysis

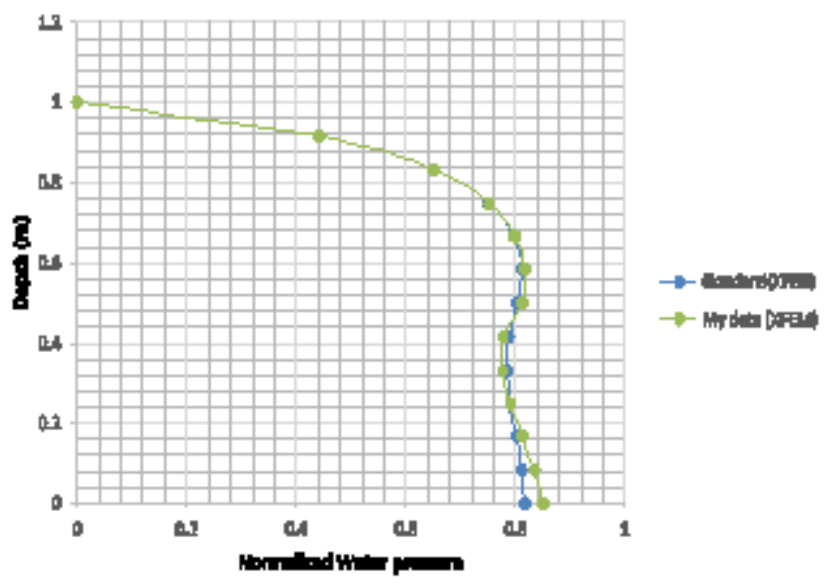

Fig. 6. Pore Water Pressure Distribution Along The Column Height

\section{CONCLUSION}

In this paper extended finite element method is utilized for modeling weak discontinuities in saturated porous media. This theory proved to be a sufficient method to capture the weak discontinuities. And helps to model the discontinuities with acceptable accuracy without necessity of implementing refined meshing.

\section{REFERENCES}

[1] K. Terzaghi, "Theoretical soil mechanics", Wiley, 1943. http://dx.doi.org/10.1002/9780470172766

[2] M. A. Biot,"General theory of three-dimensional consolidation", J Appl Phys. Vol. 12, pp. 155-64, 1941. http://dx.doi.org/10.1063/1.1712886

[3] R. De Boer, S. Kowalski, "A plasticity theory for fluid saturated porous media," International Journal of Engineering Science, vol. 21(11), pp. 1343-1357, 1983. http://dx.doi.org/10.1016/0020-7225(83)90132-5

[4] O. C. Zienkiewicz, T. Shiomi, "Dynamic behavior of saturated porous media: the generalized Biot formulation and it's numerical solution," Int J Numer Anal Methods Geomech. Vol. 8, pp. 71-96, 1984. http://dx.doi.org/10.1002/nag.1610080106

[5] A. Daneshyar, S. Mohammadi, "Strong tangential discontinuity modeling of shear bands using the extended finite element method," Computational Mechanics, Vol. 52 (5), pp. 1023-1038, 2013. http://dx.doi.org/10.1007/s00466-013-0861-8

[6] J. M. Melenk, I. Babuška, "The partition of unity finite element method: basic theory and applications," Computer Methods in Applied Mechanics and Engineering, vol.139, pp. 289-314, 1996.

http://dx.doi.org/10.1016/S0045-7825(96)01087-0

[7] I. Babuška, J. M. Melenk," The partition of unity method. International Journal for Numerical Methods in Engineering," 40:727-758, 1997. http://dx.doi.org/10.1002/(SICI)1097-0207(19970228)4 $0: 4<727::$ AID-NME86>3.0.CO;2-N

[8] T. Strouboulis, I. Babuška, K. Copps, "The design and analysis of the Generalized Finite Element Method," Computer Methods in Applied Mechanics and Engineering; vol. 181, pp. 43-69, 2000.

http://dx.doi.org/10.1016/S0045-7825(99)00072-9

[9] T. Strouboulis, K, Copps, I. Babuška, "The generalized finite element method," Computer Methods in Applied Mechanics and Engineering; vol. 190, pp. 4081-4193, 2001. http://dx.doi.org/10.1016/S0045-7825(01)00188-8

[10] N. Moes, J. Dolbow, T. Belytschko, "A finite element method for crack growth without remeshing", International Journal of Numerical Methods in Engineering, vol. 46, pp.131-150, 1999.

http://dx.doi.org/10.1002/(SICI)1097-0207(19990910)4 6:1<131::AID-NME726>3.3.CO;2-A

[11] T. Belytschko, T. Black, "Elastic crack growth in finite elements with minimal remeshing" vol. 63 (55), pp. 760-788, 1999.

[12] S. Mohammadi, Extended finite element method, Blackwell, 2007.

[13] M. Goudarzi, S. Mohammadi, "Weak discontinuity in porous media: an enriched EFG method for fully coupled layered porous media," International Journal of Numerical and Analytical Methods in Geomechanics, vol. 38, pp. 1792-1822, 2014. http://dx.doi.org/10.1002/nag.2281

[14] S. Samimi, A. Pak, "Three-dimensional simulation of fully coupled hydro-mechanical behavior of saturated porous media using Element Free Galerkin (EFG) method,' Computers and Geotechnics, 46:75-83, 2012. http://dx.doi.org/10.1016/j.compgeo.2012.06.004

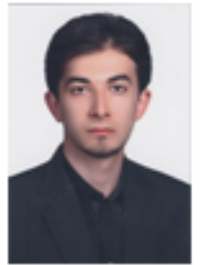

$\mathrm{He}$ is from Iran. His birthdate is 1991 /september $/ 1 \mathrm{He}$ is graduate student from Sharif University of Technology in department of civil engineering Tehran, Iran. He eamed his master in 2015 . Mr. EhsanMiksaili

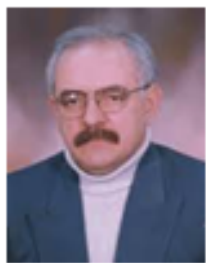

$\mathrm{HeLives}$ in Tehran. $\mathrm{He}$ is Professor at SharifUniversity of Technology, in department of civil engineering. He was graduated from Northwestem university, department of civil engineering.

Prof. M.T. Kazzmi 